\title{
Effective connectivity differences in motor network during passive movement of paretic and non-paretic ankles in subacute stroke patients
}

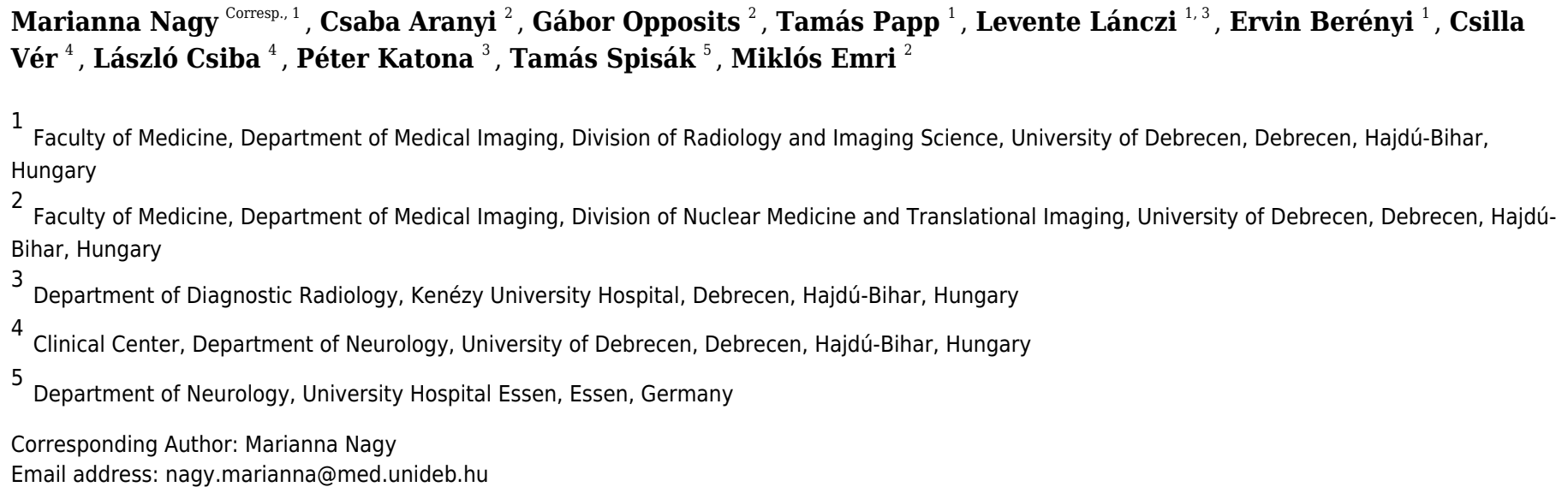

Background: A better understanding of the neural changes associated with paresis in stroke patients could have important implications for therapeutic approaches. Dynamic Causal Modeling (DCM) for functional magnetic resonance imaging ( $\mathrm{fMRI}$ ) is commonly used for analyzing effective connectivity patterns of brain networks due to its significant property of modeling neural states behind fMRI signals. We applied this technique to analyze the differences between motor networks (MNW) activated by continuous passive movement (CPM) of paretic and non-paretic ankles in subacute stroke patients. This study aimed to identify CPM induced connectivity characteristics of the primary sensory area (S1) and the differences in extrinsic directed connections of the MNW and to explain the hemodynamic differences of brain regions of MNW. Methods: For the network analysis, we used ten stroke patients' task fMRI data collected under CPMs of both ankles. Regions for the MNW, the primary motor cortex (M1), the premotor cortex (PM), the supplementary motor area (SMA) and the S1 were defined in a data-driven way, by independent component analysis. For the network analysis of both CPMs, we compared twelve models organized into two model-families, depending on the S1 connections and input stimulus modelling. Using DCM, we evaluated the extrinsic connectivity strengths and hemodynamic parameters of both stimulations of all patients. Results: After a statistical comparison of the extrinsic connections and their modulations of the "best model", we concluded that three contralateral self-inhibitions (CM1, CS1, and CSMA), one contralateral inter-regional connection ( $\mathrm{CSMA} \rightarrow \mathrm{CM} 1$ ), and one interhemispheric connection ( $\mathrm{CM} 1 \rightarrow \mathrm{iM} 1$ ) were significantly different. Our research shows that hemodynamic parameters can be 
estimated with the Balloon model using DCM but the parameters do not change with stroke. Conclusions: Our results confirm that the DCM-based connectivity analyses combined with Bayesian model selection may be a useful technique for quantifying the alteration or differences in the characteristics of the motor network in subacute stage stroke patients and in determining the degree of MNW changes. 


\section{Effective Connectivity Differences in Motor Network}

2 During Passive Movement of Paretic and Non-Paretic

3 Ankles in Subacute Stroke Patients

4

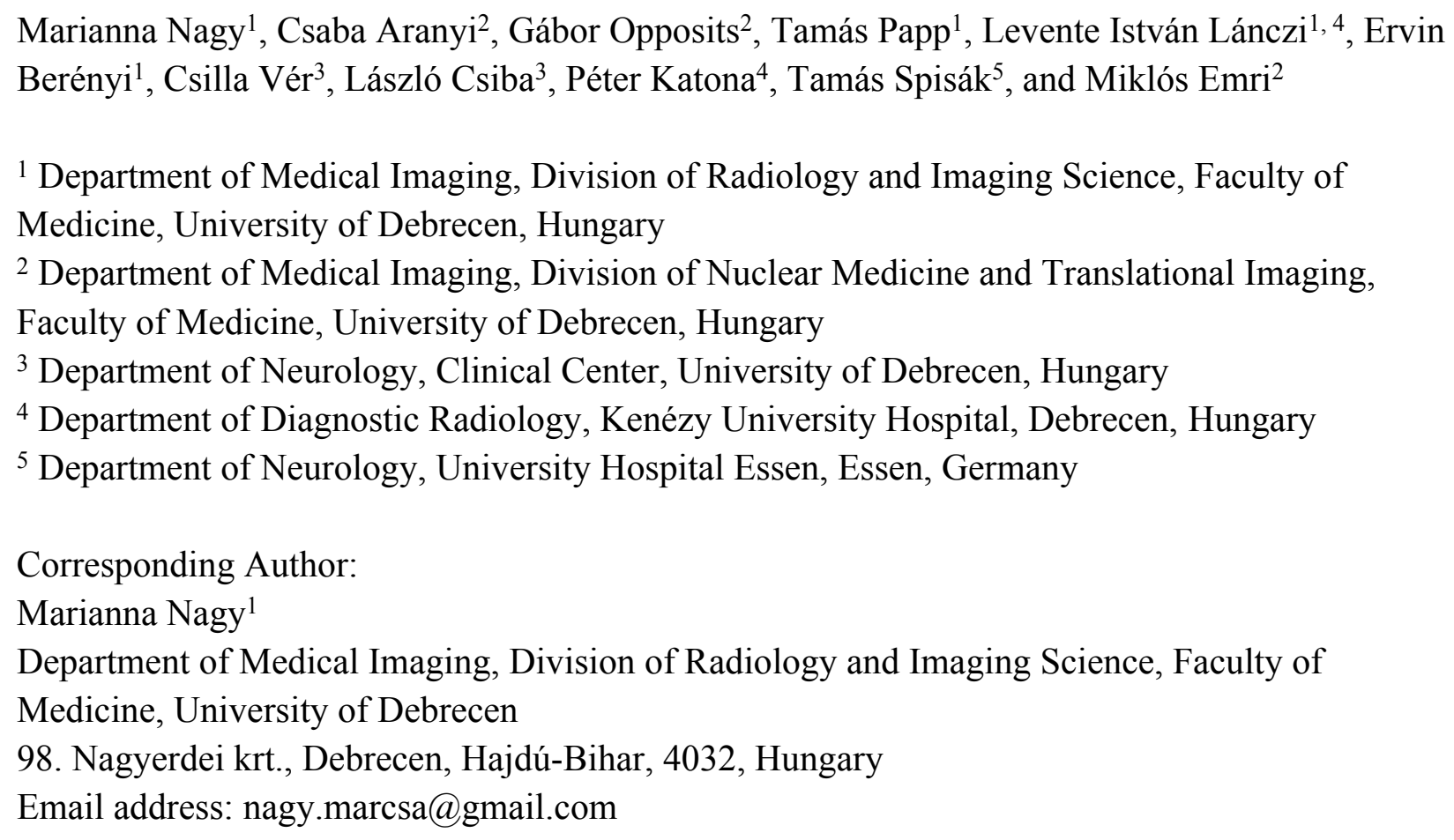

\section{Abstract}

Background: A better understanding of the neural changes associated with paresis in stroke patients could have important implications for therapeutic approaches. Dynamic Causal Modeling (DCM) for functional magnetic resonance imaging (fMRI) is commonly used for analyzing effective connectivity patterns of brain networks due to its significant property of modeling neural states behind fMRI signals. We applied this technique to analyze the differences between motor networks (MNW) activated by continuous passive movement (CPM) of paretic and non-paretic ankles in subacute stroke patients. This study aimed to identify CPM induced connectivity characteristics of the primary sensory area (S1) and the differences in extrinsic directed connections of the MNW and to explain the hemodynamic differences of brain regions of MNW. Methods: For the network analysis, we used ten stroke patients' task fMRI data collected under CPMs of both ankles. Regions for the MNW, the primary motor cortex (M1), the premotor cortex (PM), the supplementary motor area (SMA) and the S1 were defined in a datadriven way, by independent component analysis. For the network analysis of both CPMs, we compared twelve models organized into two model-families, depending on the S1 connections and input stimulus modelling. Using DCM, we evaluated the extrinsic connectivity strengthsand hemodynamic parameters of both stimulations of all patients. Results: After a statistical 
40 comparison of the extrinsic connections and their modulations of the "best model", we concluded

41 that three contralateral self-inhibitions (cM1, cS1, and cSMA), one contralateral inter-regional

42 connection ( $\mathrm{cSMA} \rightarrow \mathrm{cM} 1$ ), and one interhemispheric connection (cM1 $\rightarrow \mathrm{iM} 1$ ) were significantly

43 different. Our research shows that hemodynamic parameters can be estimated with the Balloon

44 model using DCM but the parameters do not change with stroke. Conclusions: Our results

45 confirm that the DCM-based connectivity analyses combined with Bayesian model selection

46 may be a useful technique for quantifying the alteration or differences in the characteristics of

47 the motor network in subacute stage stroke patients and in determining the degree of MNW

48 changes.

49

\section{Introduction}

51

52

53

54

55

56

57

58

59

60

61

62

63

64

65

66

67

68

69

70

71

72

73

74

75

76

77

78

79

Numerous studies have used functional magnetic resonance imaging (fMRI) to investigate neural reorganization and functional recovery after stroke (Calautti et al., 2007). fMRI univariate analysis of regional activation is valuable for understanding the regional neural substrates associated with cognitive functions (Ma et al., 2015). Using active and passive motion-based fMRI experiments (Lazaridou et al., 2013; Cheng et al., 2012) researchers have typified the poststroke motor dysfunction and the corresponding potential cerebral reorganization. In our previous therapeutic study, we examined the effects of passive movement on blood oxygen leveldependent (BOLD) responses in both hemispheres (Vér et al., 2016). Our results showed that passive movement of the paretic ankle increased BOLD responses in the contralateral pre- and postcentral gyrus, superior temporal gyrus, central opercular cortex, and in the ipsilateral postcentral gyrus, frontal operculum cortex and cerebellum.

Various brain areas are responsible for the execution of movements: the primary motor cortex (M1), supplementary motor area (SMA), and the premotor cortex (PM). The M1 controls the execution of movement by generating neural impulses (Penfield \& Boldrey, 1937) and the PM regulates the initiation of different motion patterns (Hoshi \& Tanji, 2000). The role of the SMA is also planning movement, but this is an essential brain area that is activated even when the motion is not performed, but we are only thinking about it (Tanji \& Shima, 1994). These regions create the motor network (Biswal et al., 1995) which is primarily responsible for conducting and controlling a wide variety of movements (Lam et al., 2018).

In the literature, many researchers used fMRI to investigate the connections and connectivity patterns amongst motor areas during active movement of paretic upper limb after stroke compared to controls. These studies (Rehme et al., 2011; Bajaj et al., 2016) showed that task demands alter the intensity and volume of brain activity and that these activation changes are specific to certain brain regions.

Dynamic Causal Modeling (DCM) for fMRI is commonly used for analyzing effective connectivity patterns of brain networks. The connection strength between brain regions is assessed at the underlying neuronal level rather than the observed hemodynamic level (Friston, Harrison \& Penny, 2003). In DCM connectivity is expressed in $\mathrm{Hz}$ that shows the rate at which a 
80 region's activity is mediated to another region that is directly connected to. DCM provides a fully

81 Bayesian framework to estimate the connectivity strengths of neural interactions between brain

82 regions as well as the regional self-inhibitory effects and regional hemodynamic parameters.

83 DCM considers several variables such as hemodynamic response, flow induction, activity-

84 dependent signal, change in volume and the level of deoxyhemoglobin. It uses the extended

85 Balloon model (Friston et al., 2000) to describe hemodynamic changes due to neuronal activity.

86 For studying the effective connectivity of several diseases DCM has been successfully used

87 (Seghier et al., 2010).

88 Cerebrovascular disorders may affect the shape of the hemodynamic response function (HRF) of

89 BOLD signal. In such patients, HRFs can have lower amplitudes, longer time to peak (TTP), and

90 deeper initial dips (Altamura et al., 2009; Roc et al., 2006). These observations were also

91 described in cases of retained neuronal activity (Binkofsk \& Seitz, 2004; Röther et al., 2002) and

92 in relation to altered cerebral hemodynamics (Altamura et al., 2009; Hamzei et al., 2003),

93 suggesting that decreased BOLD signals might reflect the reduction of neuronal activation or be

94 the result of neurovascular uncoupling.

95 Our study investigates whether the impairment of cerebral hemodynamics in subacute stroke

96 patients is related to changes in the BOLD signal HRF.

97 Task-dependent effective connectivity among motor cortex regions has been documented at rest

98 and during whole-hand fist closing (Grefkes et al., 2008; Nowak et al., 2008). In able-bodied

99 individuals, the strength and sign of neural coupling between motor areas is modulated by the

100 task (i.e., rest, unilateral, bilateral).

101 Investigations from the upper limb suggest that the intensity and volume of activity in the

102 primary sensory and motor cortices (S1 and M1) and in the cerebellum are sensitive to

103 movement rate (Saleh et al., 2016). In contrast with M1, S1, and the cerebellum, the effect of

104 upper extremity movement rate is less robust in the premotor and supplementary motor areas

105 (PM and SMA, respectively) (Inman et al., 2012). Less is known about the brain activation

106 pathway changes during continuous passive movement (CPM) of a lower extremity.

107 Observations from the upper limb provide a framework for understanding how brain activity

108 changes across different lower limb movement tasks. However, whether upper and lower limb

109 movements are similarly controlled with respect to rate and complexity remains unclear because

110 few studies have examined this issue during tasks of the lower limbs (Vinehout, Schmit \&

111 Schindler-Ivens, 2019). Differences in the characteristics of arm and leg movements suggest that

112 supraspinal control may also be different (Mehta et al., 2012; Cleland \& Schindler-Ivens, 2018).

113 Many DCM studies aimed to find the best model for the motor network (Volz et al., 2015;

114 Diekhoff-Krebs et al., 2017) and in most cases, the best model was the fully connected model.

115 For the upper limb, Rehme et al. (Rehme et al., 2011) investigated the temporal evolution of

116 intra- and interhemispheric connectivity during motor recovery from the acute to the early

117 chronic phase post-stroke. They analyzed 17 possible models and found the fully connected

118 model provided the best results through groups and series. In a similar study, Bajaj et al. studied

119 the connectivity pattern of the motor network in the affected and unaffected hemisphere during 
120 finger-tapping task with healthy and paretic arms (Bajaj et al., 2016). From the eight defined

121

122

123

124

125

126

127

128

129

130

131

132

133

134

135

136

137

138

139

140

141

142

143

144

145

146

147

148

149

150

151

152

153

154

155

156

157

158

159

connectivity models, they found that the full model fitted the measured data well in the affected hemisphere when the task was completed by the affected hand. In similar research, Volz et al. (Volz et al., 2015) compared the changes in motor network connectivity during upper and lower limb motion. They examined 39 models and also found that the best was the fully connected model. In contrast, we defined model families depending on the external stimulus and the connectivity patterns of the S1 region and use the Bayesian model selection technique, which provides evidence for one model over other based upon evidence ratios (i.e., Bayes factors) or differences in log evidence (Congdon, 2007).

In our previous publication (Aranyi et al., 2017), we examined potential sources of systematic motion artefacts in stroke using fMRI concentrating on those causing stimulus-correlated motion on the individual-level and separated the motion effect change on the fMRI signal from the activation-induced modification at the population level. To allow the models accounting for the sensory dimension of CPM (sensory-feedback), we added the S1 and designed model families to map its connectivity pattern to the motor system.

The aim of the study was to examine the motor network and the connectivity differences in both hemispheres during continuous passive movement (CPM) of non-paretic and paretic ankles in subacute stroke patients in the context of the neuroanatomical and functional background. We specifically focused on the role of the S1 within the network and investigated these modified networks' connectivity differences during CPM of non-paretic and paretic ankles in stroke patients. For the network analysis, we used a DCM-based Bayesian model selection to determine (1) the relationships between the S1 and the motor network and (2) to identify the brain regions that are handling the external stimulations. After the DCM model selection, we (3) investigated the extrinsic connection strength differences between the two CPMs and (4) the dissimilarity of the hemodynamic characteristics of brain regions belonging to the investigated networks.

Note that in contrast to most DCM studies, we leveraged the ability to make inferences about neuronal and hemodynamic coupling. This is particularly prescient in the context of stroke research, where lesions can produce both neuronal and neurovascular disconnections.

\section{Materials \& Methods}

\subsection{Subjects}

Ten stroke patients (mean time of stroke: 18.2 days $(\mathrm{SD}=11.4)$; mean age: 64 years $(\mathrm{SD}=7.2)$; female/male distribution: 5/5) were selected from a therapeutic study (Vér et al., 2016). Patient data, location of the lesion, and National Institutes of Health Stroke Scale (NIHSS) scores are summarized in Table 1. Each patient provided written informed consent prior to participation.

\section{Table 1.}

Due to ischemic stroke, patients had moderate or severe lower limb paresis. In our previous study (Vér et al., 2016), we applied the NIHSS to assess the functional state and the severity of 
160 the stroke in the patients. NIHSS is one of the most commonly used scales in the clinical field;

161

162

163

164

165

166

167

168

169

170

171

172

173

174

175

176

177

178

179

180

181

182

183

184

185

186

187

188

189

190

191

192

193

194

195

196

197

198

199

increasing scores indicate less functional states of the patients (Brott et al., 1989). Table 2 shows the definitions of mild, moderate and severe paresis.

The inclusion criteria were ischemic stroke confirmed via clinical investigations and computed tomography (CT). We selected subacute stage patients who were less than 30 days post-stroke. Pavlova et al. defined acute stage 1 day to 1 week, subacute stage 1 week to 1 month and chronic stage is more than 1 month after stroke (Pavlova et al., 2019).

Ability to cooperate during the MRI measurement was an inclusion criterion.

This study was approved by the Regional and Institutional Research Ethics Committee of the Scientific Committee of the University of Debrecen, Clinical Center (DE OEC RKEB / IKEB 3772-2012; DE OEC RKEB / IKEB 3983-2013).

Table 2.

2.2. Functional Magnetic Resonance Imaging

Functional and structural images were acquired at the Kenézy University Hospital, Debrecen, Hungary using a 1.5 Tesla Siemens Magnetom Essenza MR scanner. After the 3D T1-weighted MP-RAGE structural image acquisition $(\mathrm{TE}=4.73 \mathrm{~ms}, \mathrm{TR}=1540 \mathrm{~ms}$, TI=800 ms, flip angle=15 slices with $0.9 \times 0.9 \times 0.9 \mathrm{~mm}$ voxels) two series of functional whole-brain images were obtained for every subject using a BOLD contrast sensitive gradient-echo echo-planar sequence (TE=42 $\mathrm{ms}$, flip angle $=90$, in-plane resolution $=3 \times 3 \mathrm{~mm}$; volume $\mathrm{TR}=4000 \mathrm{~ms}$, axial slice thickness $=3.3$ $\mathrm{mm}$ ). The two series involved the task of passive movement of the left or the right ankle separately. Both series comprised 100 volumes that lasted 400 seconds containing 40 s-long blocks of active and inactive periods. Following an initial resting period, active and inactive blocks alternated throughout the session. During the inactive blocks, no stimulus was applied, whereas in the active blocks, slow $(\sim 1 \mathrm{~Hz})$ continuous passive movement $(\mathrm{CPM})$ of the left or right ankle was performed by the physiotherapist. The legs and the hip of the patients were fastened to the bed.

\subsection{Data Analysis}

\subsubsection{Image Preprocessing}

Before preprocessing, the left and the right sides of the structural and functional images of patients with left hemispheric lesion were mirrored. This step facilitated a pooled populationlevel statistical analysis for all the patients and prevented the need to split the population into two cohorts depending on the side of the stroke.

The image processing pipeline followed the steps used in previous DCM studies regarding motor control in stroke patients (Grefkes et al., 2008; Saleh et al., 2016). To erase non-brain areas from

Peer] reviewing PDF | (2019:11:43479:1:1:NEW 9 Mar 2020) 
200 the functional and structural scans, the brain extraction tool (BET) of FSL was used (Smith, 201 2002). The high resolution, brain extracted T1 images were spatially standardized to match the 202 symmetric template of MNI152 space (Grabner et al., 2006) using the linear and non-linear 203 registration utilities of the FSL package (Jenkinson et al., 2012). We verified the correctness of 204 the image transformation results via visual inspection.

205 For further analysis, the first three volumes of each functional dataset were excluded to avoid the 206 equilibrium effect of T1 images. The fMRI images were motion-corrected with the MCFLIRT 207 utility of FSL software (Jenkinson et al., 2000). The same software was applied to extract the six 208 motion parameters (three rotations and three translation components of rigid body

209 transformations). The fMRI data were then co-registered to the extracted anatomical brain image 210 and spatially transformed to MNI152 space using the deformation field obtained from the T1 211 standardization process.

212 On the functional images, we applied an isotropic Gaussian smoothing with $8 \mathrm{~mm}$ full width half 213 maximum (FWHM).

214

\subsubsection{Independent Component Analysis}

After independent component analysis (ICA) (Jung et al., 2001), we selected components

218 corresponding to motor network areas (M1, PM, and SMA) after visual inspection of the statistical Student-t maps and characteristic time-series of the components on individual level.

220 We completed this search by identifying the primary somatosensory cortex (S1) to examine the effects of the touching of the patients' legs by the physiotherapist during the passive movement task. S1 is responsible for delivering information about the stimulation of the skin, the intestinal

223 mucous membrane of the internal organs, and about the position of the body parts (Borich et al., 2015). We chose the highest $z$ value within each cluster as the centre of the regions used in DCM analysis.

225

\subsection{Dynamic Causal Modeling}

227 DCM is used to describe the causative structure of coupled dynamic systems. Using the observable phenomenon of functional connectivity, which can be measured by correlations, for example, and a special model that describes the observed statistical dependencies, we can map the relationships between different brain areas. We used a stochastic variant of the DCM algorithm (Li et al., 2011) that accommodates endogenous or random fluctuations in hidden neuronal states. This variant is useful in most cases because modelling state-noise is challenging. Allowing for random neuronal fluctuations results in an estimation scheme more robust to model misspecification. A drawback of this method compared to the original deterministic DCM is that the parameter estimation procedure, also referred to as model inversion is much more computationally demanding. To limit the parameters with which connection strength can be estimated, we restricted our DCM model space to 239 combine extrinsic connections and direct stimulating effects on brain regions. 
240

241

242

243

244

245

246

247

248

249

250

251

252

253

254

255

256

257

258

259

260

261

262

263

264

265

266

267

268

269

270

271

272

273

274

275

276

277

278

\subsection{Systematic Building of Model-Space}

For building a DCM model-space for both fMRI sessions, we created bilateral models in relation to the contralateral and ipsilateral brain hemispheres to the moved limb. For finding the most probable connectivity architectures in the motor network based on the measured data, we defined a two-sided base model: the extrinsic (i.e., between-region) directed connectivity between PM, SMA, and M1 regions were fully connected within both hemispheres, and inter-hemispheric connections were set between the M1s and SMAs (Figure 1A). To investigate the connections between $\mathrm{S} 1$ and other regions within both sides of the movement, we defined four possible network extensions: (1) S1 does not connect with PM, SMA and M1; (2) S1 only connects to M1; (3) S1 only connects to PM; and (4) S1 connects to PM and M1 (Figure 1B, columns). For checking the target regions of the external stimulus at the contralateral side of movement, we considered three functional variations: (1) the stimulus directed to the PM and S1; (2) the stimulus directed to the PM, S1, and M1; and (3) the stimulus directed to the S1 and M1 (Figure $1 \mathrm{~B}$, rows). Combining these possibilities, we established a model space comprising $4 \times 3=12$ model variants (Model1, Model2, - ..., Model12), which were arranged into a matrix form (Figure 1B), to emphasize the factorial structure of this model space. The row view of this arrangement represents the first model family set containing three model families (FS11, FS12, and FS13) concerning the direct stimulus effect variants. The column view of this matrix shows the second model family set consisting of four families according to the S1 connection arrangements (Fstim1, Fstim2, Fstim3, and Fstim4).

Figure 1C shows an example of the combination of the base model and Model 4.

\section{Figure 1.}

Statistical analysis of between-subject (i.e., group) effects was performed under the winning model using (nonparametric) classical inference. The winning model was identified by pooling the evidence for different models over all subjects studied.

\subsection{Comparison of Model Families and Model Selection}

We used a Bayesian model comparison (BMC) (Penny et al., 2010) technique on both model family sets to assess the most probable combination of these connectivity parameters based on the fMRI patient. BMC focuses on a model structure that is created by defining and building a model space. Model space usually means a set of models, where each model assigns specific endogenous connections. The BMC procedure determines the model that best describes how the data are generated by computing the expected and exceedance probability of each model (Penny et al., 2004; Stephan et al., 2009). Expected probability shows the probability that a given model generated the measured data. Exceedance probability indicates the probability that a given model is more likely than any other models in the comparison. After the two BMC calculations, we 
279 identified the best model family from both family sets, and we considered the mutual model of 280 these selected families as the winning model, which can be described by the measured data.

281

282

283

284

285

286

287

288

289

290

291

292

293

294

295

296

297

298

299

300

301

302

303

304

305

306

307

308

309

310

311

312

313

314

315

316

317

318

\subsection{Hemodynamic Parameters}

Neuronal activity leads to fMRI data by a dynamic process characterized by a Balloon model (Buxton et al., 1998) and BOLD signal model (Stephan et al., 2007) for each brain region. This defines how changes in neuronal activity cause changes in blood oxygenation that are measured with fMRI.

DCM not only investigates the connection system during the model inversion but also estimates the regional hemodynamic parameters of the Balloon model: hemodynamic signal decay (D), transit time (T), and the ratio of intra- and extra-vascular components of gradient echo-signal (E) (Stephan et al., 2007). An increase in the signal decay (D) reduces the regional cerebral blood flow (rCBF) response to any input and suppresses the undershoot. The effect of increasing transit time $(\mathrm{T})$ is to slow down the dynamics of the BOLD signal regarding to the flow changes. The parameter $\mathrm{E}$ reflects the efficacy of the following synaptic activity to generate the signal and the potency of the stimulus in obtaining a neuronal response (Friston et al., 2000).

In this study, we examined the hemodynamic parameters in subacute stroke patients calculated by the DCM of the winning model for statistical analysis.

\subsection{Statistical Analysis}

Because the non-paretic and paretic ankle CPMs induced lateralized brain activations, we had to relabel the brain regions' names in the point of view of their laterality before statistical analysis. Therefore, we used the ipsi-, and contralateral nomenclature (noted by $i$ and c prefixes, respectively) as Rehme and Saleh (Rehme et al., 2011; Saleh et al., 2016) used for denoting the position of brain regions relative to the two types of stimuli, i.e., cM1, cSMA, cPM, cS1 and iM1, iSMA, iPM, iS1. This allowed us to compare the connectivity strengths and hemodynamic parameters of the activated (or passive) regions during the CPMs of the paretic and the nonparetic ankles.

The expected distributions of the self-inhibition and inter-regional connections are different, because self-connections are log scaling parameters of a negative a priori value $(-0.5 \mathrm{~Hz})$ to ensure system stability in DCM, while extrinsic connections are not scaled and have a prior expectation of $0 \mathrm{~Hz}$. Thus, we separately investigated the normality of data using the ShapiroWilk tests. Because these tests showed non-normality, we performed a mass of Monte-Carlobased exact permutation tests to statistically characterize the stimulus-related differences between the paretic and non-paretic ankles in the elements of the endogenous connectivity matrix (matrix A) and the parameters of modulatory effects (matrix B). Similarly, neither external stimulus strength (matrix $\mathrm{C}$ ) nor hemodynamic parameters were found normal distribution, so we applied the same comparison technique for these data.

To correct for multiple comparisons, we applied the false discovery rate (FDR) for adjusting pvalues (Benjamini \& Yekutieli, 2001) calculated by the statistical tests. 


\section{Results}

321

322

323

324

325

326

327

328

329

330

331

332

333

334

335

336

337

338

339

340

341

342

343

344

345

346

347

348

349

350

351

352

353

354

355

356

357

358
Using BMC selection, we found that during the CPM task, the S1 connection with the M1 and $\mathrm{PM}\left(\mathrm{F}^{\mathrm{S} 1}{ }_{4}\right.$ family) was the most-likely network topology with 0.784 expected probability and 0.998 exceedance probability (Figure 2A/B). For direct effect model family selection, we observed that the stimulus driving S1, M1 and PM ( $\mathrm{F}^{\text {stim }}{ }_{2}$ family) was the most probable model with 0.845 expected probability and 0.999 exceedance probability (Figure 2C/D). Using these results, we selected Model11 (Figure 3) as the winning model for the statistical analysis.

Figure 2.

Figure 3.

As the Shapiro-Wilk tests resulted in $\mathrm{p}<0.001$ values in all datasets, we used Monte-Carlobased exact permutation tests in all connectivity and hemodynamic parameter comparisons. Following the statistical analysis of the endogenous connectivity matrix of the winning model (Model 11), we summarized the results in Table 3.

\section{Table 3.}

Using the FDR-corrected p-values (pFDR), we concluded that three contralateral self-inhibitions $(\mathrm{cM} 1, \mathrm{cS} 1$, and cSMA), one contralateral inter-regional connection (cSMA $\rightarrow \mathrm{cM} 1$ ), and one interhemispheric connection ( $\mathrm{cM} 1 \rightarrow \mathrm{iM} 1)$ were significantly different during the comparison of the two CPMs (Figure 4).

\section{Figure 4.}

Comparing the mean values, we showed that the paretic CPM caused stronger self-inhibition in $\mathrm{cM} 1$ and $\mathrm{cS} 1$ and weaker self-inhibition in SMA (Figures 5A-C). The connection between cSMA and cM1 dramatically changed (Figure 5D ): during the non-paretic CPM, the cSMA excited the neural activity of $\mathrm{cM} 1$, which in turn inhibited the paretic stimulus. The interhemispheric $\mathrm{cM} 1 \rightarrow \mathrm{iM} 1$ connection shown showed stronger excitation during the paretic CPM in relative to the non-paretic ankle's movement (Figure 5E).

Figure 5.

The statistical examination did not show any significant differences neither in the analysis of the external stimulus (matrix C) or in the Balloon model parameter comparisons. 


\section{Discussion}

360

361

362

363

364

365

366

367

368

369

370

371

372

373

374

375

376

377

378

379

380

381

382

383

384

385

386

387

388

389

390

391

392

393

394

395

396

397

398

In this work, we examined the connection topology of the S1-extended motor network and investigated the differences in passive-movement related effective connectivity between the paretic and non-paretic limbs during CPM in subacute stroke patients. As the exact effective connectivity structure of the motor network in stroke is not well known, we applied an fMRIbased model-search procedure to identify the model family that best fits the motor network (Kahan \& Foltynie, 2013; Penny et al., 2010). According to previous research, in this study, we used the DCM-based effective connectivity technique to describe the motor network properties during the applied CPM stimulations. We chose this method because it helps to understand the causal architecture of the modelled networks by considering the temporal variation in the neural activity estimated by the BOLD signal. We have used a conservative approach to compare extrinsic connectivity between paretic and non-paretic stimulations of the investigated patient group. This nonparametric approach to classical inference at the between-subject level is conservative because it tests for differences in each connection separately (using FDR to adjust for multiple comparisons). We could have used a multivariate test (e.g., canonical covariates analysis). This would have been more sensitive and would have highlighted significant stimulation differences. However, we would not have been able to assign a unique FDR's q value to each connection. Finally, it should be noted that more recent analyses of betweensubject effects on effective connectivity in dynamic causal modelling would normally use parametric empirical Bayes (Friston et al., 2016; Kass \& Steffey, 1989). However, our classical inference is sufficient for our purposes - and represents a simple way of accommodating between-subject random effects.

For model selection, we defined two model families containing four and three models according to the model-combinations of the S1 connections and the external stimuli modelling, respectively. The applied BMC selection showed that the external sensory stimulation bound to the S1 and PM regions, and the S1 had causal connections only with M1 and PM. A modelfamily-based Bayesian model selection was also applied by Saleh et al. (Saleh et al., 2016), who examined the interactions between regions that may modulate the activation of the ipsilesional motor cortex during visual mirror feedback of unaffected hand movement in stroke patients. They also identified a non-fully connected topological scheme as the best model. After statistical comparison of the extrinsic connections of the winning model during the nonparetic and paretic CPM, we concluded that three contralateral self-inhibitions (cM1, cS1, and $\mathrm{cSMA}$ ), one contralateral inter-regional connection ( $\mathrm{cSMA} \rightarrow \mathrm{cM} 1)$, and one interhemispheric connection $(\mathrm{cM} 1 \rightarrow \mathrm{iM} 1)$ were significantly different. Some neuroimaging studies reported that during the movement of stroke-affected paretic limbs, a significant neural activity could be observed in the regions of the contralesional hemisphere (Calautti et al., 2007; Ma et al., 2015; Lazaridou et al., 2013; Badillo, Vincent \& Ciuciu, 2013; Ward et al., 2003). Nowak et al. demonstrated that overactivity in contralesional M1 occurs early after stroke, affecting the improvement of these brain regions after the vascular incident Nowak et al., 2008). Grefkes et al.

Peer) reviewing PDF | (2019:11:43479:1:1:NEW 9 Mar 2020) 
399

400

401

402

403

404

405

406

407

408

409

410

411

412

413

414

415

416

417

418

419

420

421

422

423

424

425

426

427

428

429

430

431

432

433

434

435

436

437

438

also showed that the time after stroke is an essential factor influencing brain motor network analysis (Grefkes et al., 2008).

The human motor network in the hemispheres contains several parts, including the primary motor cortex (M1), supplementary motor area (SMA) and premotor cortex (PMC), which territories show ordered representation of the human body, called as the somatotopic representation. All the parts of the above territories send collateral fibres into the another hemispheres through the corpus callosum, which fibres reaches the same somatotopic territories (homotopic regions) (van den Heuvel \&, Hulshoff, 2010). However, anatomical connectivity shows difference between the upper and lower extremities, e.g. the activation of $\mathrm{M} 1_{\text {hand }}$ inhibit the contralateral $\mathrm{M} 1_{\text {hand }}$ territory, whereas the activation of $\mathrm{M} 1_{\text {foot }}$ accused facilitatory coupling in the contralateral hemisphere (Volz et al., 2015). The SMA and PMC have strong input into the M1, therefore this adjacent territories can enhance the motor output of M1 pyramidal cells (Dancause et al., 2005; Dum et al., 2005). The primary somatosensory cortex (S1) also able to modify peripheral movements independently (a small part of the corticospinal tract originating from the S1) or dependently from M1 in healthy patients or in stroke survivors. Here the anatomical basis is the U-fibers which caused a strong connectivity between the neighbouring gyruses (Borich et al., 2015). According to human data the increased peripheral somatosensory inflow helps in the reorganization of M1 after stroke (Borich et al., 2015). This connection between the M1 and S1, the literature uses the sensorimotor synchronization (SMS).

The different SMA-M1 connection strength is a common finding in many motor-based DCM studies (Ward et al., 2003; Wang et al., 2016; Moulton et al., 2017). This connectivity, present for simple and complex tasks alike, has been attributed to the respective role of the SMA and M1 in voluntary upper limb movements (Moulton et al., 2017). Pool et al. (Pool et al., 2013) showed that movements at higher frequencies are linked with a linear increase in neural coupling strength, especially from contralateral SMA to contralateral M1. This result shows that SMA cooperates with variations in hand motor performance. Based on this and our results, we concluded that the same alteration occurs in the case of the lower limb. The order of magnitude of the contralateral SMA $\rightarrow \mathrm{M} 1$ connection was different in the two CPMs: the SMA increased the neural activity of M1 by $0.085 \mathrm{~Hz}$ (sd: $0.0496 \mathrm{~Hz}$ ) during the non-paretic side activation, this effect altered to $-0.0053 \mathrm{~Hz}$ (sd: $0.0845 \mathrm{~Hz}$ ) in the opposite CPM, which means that this interaction occurs only in the non-paretic case. Diekhoff-Krebs et al. (Diekhoff-Krebs et al., 2017) tested the hypothesis that interindividual variability in behavioural responses to excitatory repetitive transcranial magnetic stimulation (rTMS) in stroke patients and healthy control group is related to interindividual differences in the network connections of the stimulated region. Their results revealed that a stronger connection exists between the SMA and the M1 regions before intermittent theta-burst stimulation (iTBS) intervention at the affected arm in patients with better motor performance. Our results support this statement because we also showed a strong relationship between SMA and M1 regions.

The $50 \%$ stronger cM1-iM1 interaction $(0.2761 \mathrm{~Hz}$ vs $0.1705 \mathrm{~Hz})$ during the paretic CPM may indicate a partial adaptive compensation for the injured motor cortex by the non-affected M1 
439 after stroke. Most previous studies examined the functions of the upper limb after stroke. Grefkes

440 et al. showed that the inhibitory influences on movements of the paretic hand from the

441 contralesional to the ipsilesional M1 correspond with the degree of motor disability (Grefkes et

442 al., 2008). They recommended that the motor loss of patients with a single subcortical lesion is

443 connected to pathological interhemispheric interactions between the main motor regions. This

444 can be explained why we found a difference between interhemispheric connections. The

445 observed connectivity differences suggesting that the differences are region-specific, a residual

446 uncertainty as to localization remains, that is deep-rooted to cytoarchitectonic probability maps.

447 Functional connectivity studies have been conducted in stroke yet, the changes in tissue

448 composition at the site of the lesion is at various stages of necrosis and gliosis, affecting the

449 BOLD signal (Frias et al, 2018). It cannot be excluded that the change in tissue composition

450 might have an effect on functional connectivity. Our results demonstrate that stroke can affect

451 the functional connectivity of regions distant to the infarct, specifically S1, potentially further

452 compromising motor performance.

453 We showed that the hemodynamical parameters (Balloon model's D, T, and E) of the regions of

454 the motor networks were statistically similar during the two stimulations. This result suggests

455 that the detected differences in the connection strengths originated from real neural activity, and

456 the hemodynamic change had no confounding effect during the measurements.

457 Stroke recovery is a complex mechanism that possibly the results of substitution, compensation

458 of functions and combination of restoration (Hara, 2015). Many studies addressed the recovery

459 of motor skills after an intervention procedure, and it is well documented that the healthy brain

460 regions take over the functioning of the damaged areas (Jiang, Xu \& Yu, 2013; Brown et al.,

461 2009). In a study on motor recovery following rehabilitation, Arya et al. (Arya et al., 2011)

462 proposed that the recovery could be compensatory motor recovery or real motor recovery, which

463 occurs when different connections that are unharmed send instructions to the same damaged

464 muscles to perform the motor orders. Neuronal reorganization and plasticity after a stroke takes

465 place during the first 6 months following stroke and involve brain regions distant to the affected

466 area (Li, 2017). According to Zeiler et al. after ischemic stroke, both spontaneous and

467 intervention-mediated recovery from impairment is maximal within 1-3 months (Zeiler et al.,

468 2013). Therefore it is difficult to interpret the outcomes of rehabilitative studies in human stroke 469 patients (Hara, 2015).

470

471

472 Limitations and conclusion

473

474 One limitation of the present study concerns the small sample size. We found significant changes

475 in effective connectivity despite the low number of patients. Furthermore, the difference between

476 left- or right-sided injuries or the anatomical regions of structural damage on effective

477 connectivity could be better investigated in studies with more extended groups. 
478 Another limitation of this study is related to spontaneous plasticity recovery which takes place in 479 the early post-stroke period. Physiotherapy strategies used during the recovery process affect 480 spontaneous neuroplasticity. According to further studies, the optimal timing to begin 481 rehabilitation after stroke is still not known and it is highly possible that early intervening to 482 impacts cortical reorganization in a beneficial way (Coleman at al., 2017; Cassidy \& Cramer, 483 2017).

484 In summary, our results confirm that the DCM-based connectivity analyses combined with 485 Bayesian model selection may be a useful technique for quantifying the alteration or differences 486 in the characteristics of the motor network in subacute stage stroke patients and in determining 487 the degree of MNW changes. However, in stroke, the number of patients who can be involved in 488 these types of fMRI studies is a severely limiting factor, yet.

489

490

491

492

493

494

495

496

497

498

499

500

501

Aranyi C., Opposits G., Nagy M., Berényi E., Vér C., Csiba L., Katona P., Spisák T., Emri M.

502 Population-Level Correction of Systematic Motion Artifacts in fMRI in Patients with Ischemic

503

504 Stroke. 2017. J. Neuroimaging 27: 397-408. DOI: 10.1111/jon.12408.

\section{Acknowledgements}

This research was supported by the National Brain Research Program (2017-1.2.1-NKP-201700002). There was no additional external funding received for this study.

\section{References}

Altamura C., Reinhard M., Vry M.S., Kaller C.P., Hamzei F., Vernieri F., Rossini P.M., Hetzel A., Weiller C., Saur D. 2009. The longitudinal changes of BOLD response and cerebral hemodynamics from acute to subacute stroke. A fMRI and TCD study. BMC Neurosci. 10: 151. DOI: $10.1186 / 1471-2202-10-151$.

505

Arya K.N., Pandian S., Verma R., Garg R.K. 2011. Movement therapy induced neural

506 reorganization and motor recovery in stroke: a review. J. Bodyw. Mov. Ther. 15: 528-37. DOI:

507

508

509 10.1016/j.jbmt.2011.01.023.

510

511

512 Bajaj S., Housley S.N., Wu D., Dhamala M., James G.A., Butler A.J. 2016. Dominance of the 513 Unaffected Hemisphere Motor Network and Its Role in the Behavior of Chronic Stroke

514 Survivors. Front. Hum. Neurosci. 10: 650. DOI: 10.3389/fnhum.2016.00650.

515

516 Benjamini Y., Yekutieli D. 2001. The control of the false discovery rate in multiple testing under

517 dependency. Annals of Statistics 29, 1165-1188. DOI: 10.2307/2674075. 
518

519

520

521

522

523

524

525

526

527

528

529

530

531

532

533

534

535

536

537

538

539

540

541

542

543

544

545

546

547

548

549

550

551

552

553

554

555

556

557

Binkofsk F., Seitz R.J. 2004. Modulation of the BOLD-response in early recovery from sensorimotor stroke. Neurology 63: 1223-9.

Biswal B., Yetkin F.Z., Haughton V.M., Hyde J.S. 1995. Functional connectivity in the motor cortex of resting human brain using echo-planar MRI. Magn. Reson. Med. 34: 537-41.

Bonakdarpour B., Beeson P.M., DeMarco A.T., Rapcsak S.Z. 2015. Variability in blood oxygen level dependent (BOLD) signal in patients with stroke-induced and primary progressive aphasia. Neuroimage Clin. 8: 87-94. DOI: 10.1016/j.nicl.2015.03.014.

Bonakdarpour B., Parrish T.B., Thompson C.K. 2007. Hemodynamic response function in patients with stroke-induced aphasia: Implications for fMRI data analysis. Neuroimage 36: $322-$ 331. DOI: 10.1016/j.neuroimage.2007.02.035.

Borich M.R., Brodie S.M., Gray W.A., Ionta S., Boyd L.A. 2015. Understanding the role of the primary somatosensory cortex: Opportunities for rehabilitation. Neuropsychologia 79: 246-55. DOI: 10.1016/j.neuropsychologia.2015.07.007.

Brott T., Adams H.P. Jr, Olinger C.P., Marler J.R., Barsan W.G., Biller J., Spilker J., Holleran R., Eberle R., Hertzberg V., Rorick M., Moomaw C.J., Walker M. 1989. Measurements of acute cerebral infarction: a clinical examination scale. Stroke 20: 864-870.

Brown C.E., Aminoltejari K., Erb H., Winship I.R., Murphy T.H. 2009. In vivo voltage-sensitive dye imaging in adult mice reveals that somatosensory maps lost to stroke are replaced over weeks by new structural and functional circuits with prolonged modes of activation within both the peri-infarct zone and distant sites. J. Neurosci. 29: 1719-34.

DOI:https://doi.org/10.1523/JNEUROSCI.4249-08.2009.

Buxton R.B., Wong E.C., Frank L.R. 1998. Dynamics of blood flow and oxygenation changes during brain activation: the balloon model. Magn. Reson. Med. 39, 855-864.

Calautti C., Naccarato M., Jones P.S., Sharma N., Day D.D., Carpenter A.T., Bullmore E.T., Warburton E.A., Baron J.C. 2007. The relationship between motor deficit and hemisphere activation balance after stroke: a 3 T fMRI study. Neuroimage 34: 322-331.

Cassidy J.M., Cramer S.C. 2017. Spontaneous and Therapeutic-Induced Mechanisms of Functional Recovery After Stroke. Transl Stroke Res. 8: 33-46. DOI: 10.1007/s12975-016-04675 . 
558 Cheng L., Wu Z., Fu Y., Miao F., Sun J., Tong S. 2012. Reorganization of functional brain 559 networks during the recovery of stroke: a functional MRI study. Conf. Proc. IEEE Eng. Med.

560 Biol. Soc. 4132-5. DOI: 10.1109/EMBC.2012.6346876.

561

562 Cleland B.T., Schindler-Ivens S. 2018. Brain Activation During Passive and Volitional Pedaling 563 After Stroke. Motor Control. 17: 1-29. DOI: 10.1123/mc.2017-0059.

564

565 Coleman E.R., Moudgal R., Lang K., Hyacinth H.I., Awosika O.O., Kissela B.M., Feng W.

566 2017. Early Rehabilitation After Stroke: a Narrative Review. Curr Atheroscler Rep. 19:59. DOI:

567 10.1007/s11883-017-0686-6.

568

569

Congdon P. 2007. Bayesian Statistical Modelling. Wiley.

570

571

Dąbrowski J., Czajka A., Zielińska-Turek J., Jaroszyński J., Furtak-Niczyporuk M., Mela A.,

572 Poniatowski Ł.A., Drop B., Dorobek M., Barcikowska-Kotowicz M., Ziemba A. 2019. Brain

573 Functional Reserve in the Context of Neuroplasticity after Stroke. Neural Plast. 27;

574 2019:9708905. DOI: 10.1155/2019/9708905.

575

576 Dancause N., Barbay S., Frost. S.B., Plautz E.J., Chen D., Zoubina E.V., Stowe A.M., Nudo R.J.

577 2005. Extensive cortical rewiring after brain injury. J. Neurosci. 25, 10167-10179. DOI:

578 10.1523/JNEUROSCI.3256-05.2005.

579

580

Diekhoff-Krebs S., Pool E.M., Sarfeld A.S., Rehme A.K., Eickhoff S.B., Fink G.R., Grefkes C.

581 2017. Interindividual differences in motor network connectivity and behavioral response to iTBS

582 in stroke patients. Neuroimage Clin. 15: 559-571. DOI: 10.1016/j.nicl.2017.06.006.

583

584

Dum R.P., Strick P.L. 2005. Frontal lobe inputs to the digit representations of the motor areas on

585 the lateral surface of the hemisphere. J. Neurosci. 25, 1375-1386. DOI:

586

587

588 10.1523/JNEUROSCI.3902-04.2005.

589

Frías I., Starrs F., Gisiger T., Minuk J., Thiel A., Paquette C. Interhemispheric connectivity of

590 primary sensory cortex is associated with motor impairment after stroke. Sci. Rep. 2018, 8:

591

592 12601. DOI: 10.1038/s41598-018-29751-6.

593

Friston K.J., Harrison L., Penny W. 2003. Dynamic causal modelling. Neuroimage 19: 1273302.

594

595

Friston K.J., Mechelli A., Turner R., Price C.J. 2000. Nonlinear responses in fMRI: the Balloon 596 model, Volterra kernels, and other hemodynamics. Neuroimage 12: 466-77.

597 
598 Friston K.J., Litvak V., Oswal A., Razi A., Stephan K.E., van Wijk B.C.M., Ziegler G., Zeidman 599 P. 2016. Bayesian model reduction and empirical Bayes for group (DCM) studies. Neuroimage. 600 128: 413-431. DOI: 10.1016/j.neuroimage.2015.11.015.

601

602 Grabner G., Janke A.L., Budge M.M., Smith D., Pruessner J., Collins D.L. 2006. Symmetric 603 atlasing and model based segmentation: an application to the hippocampus in older adults. Med. 604 Image Comput. Comput. Assist. Interv. 9: 58-66. DOI: 10.1007/11866763_8.

605

606 Grefkes C., Nowak D.A., Eickhoff S.B., Dafotakis M., Küst J., Karbe H., Fink GR. 2008.

607 Cortical connectivity after subcortical stroke assessed with functional magnetic resonance

608 imaging. Ann Neurol. 63: 236-46. DOI: 10.1002/ana.21228.

609

610

611

612

613

614

615

616

617

618

619

620

621

622

623

624

625

626

627

628

629

630

631

Hamzei F., Knab R., Weiller C., Rother J. 2003. The influence of extra-and intracranial artery disease on the BOLD signal in fMRI. Neuroimage 20: 1393-9.

Hara Y. 2015. Brain Plasticity and Rehabilitation in Stroke Patients. Journal of Nippon Medical School 82: 4-13. DOI: 10.1272/jnms.82.4.

He S.Q., Dum R.P., Strick P.L. 1993. Topographic organization of corticospinal projections from the frontal lobe: motor areas on the lateral surface of the hemisphere. J Neurosci. 13: 952-80.

Hoshi E., Tanji J. 2000. Integration of target and body-part information in the premotor cortex when planning action. Nature 408: 466-470.

Inman C.S., James G.A., Hamann S., Rajendra J.K., Pagnoni G., Butler A.J. 2012. Altered resting-state effective connectivity of fronto-parietal motor control systems on the primary motor network following stroke. NeuroImage 59: 227-37. DOI: 10.1016/j.neuroimage.2011.07.083.

Jaillard A., Martin C.D., Garambois K., Lebas J.F., Hommel M. 2005. Vicarious function within the human primary motor cortex? A longitudinal fMRI stroke study. Brain 128: 1122-38.

632

633

Jenkinson M., Bannister P., Brady M., Smith S. 2000. Improved Optimisation for the Robust and Accurate Linear Registration and Motion Correction of Brain Images. NeuroImage 17: 825-841. DOI: $10.1016 / \mathrm{s} 1053-8119(02) 91132-8$.

634

635

636 Jiang L., Xu H., Yu C. 2013. Brain connectivity plasticity in the motor network after ischemic 637 stroke. Neural Plast. 924192. DOI: 10.1155/2013/924192. 
638

639

640

641

642

643 Kahan J., Foltynie T. 2013. Understanding DCM: Ten simple rules for the clinician. NeuroImage

644

645

646

647

648

649

650

651

652

653

654

655

656

657

658

659

660

661

662

663

664

665

666

667

668

669

670

671

672

673

674

675

Jung T.P., Makeig S., McKeown M.J., Bell A.J., Lee T.W., Sejnowski T.J. 2001. Imaging Brain Dynamics Using Independent Component Analysis. Proc. IEEE Inst. Electr. Electron Eng. 89:

1107-1122. DOI: 10.1109/5.939827. 83: 542-549. DOI: 10.1016/j.neuroimage.2013.07.008.

Kass R.E., Steffey D. 1989. Approximate Bayesian Inference in Conditionally Independent Hierarchical Models (Parametric Empirical Bayes Models). Journal of the American Statistical Association 407: 717-726.

Lam T.K., Dawson D.R., Honjo K., Ross B., Binns M.A., Stuss D.T., Black S.E., Chen J.J., Levine B.T., Fujioka T., Chen J.L. 2018. Neural coupling between contralesional motor and frontoparietal networks correlates with motor ability in individuals with chronic stroke. J. Neurol. Sci. 384: 21-29. DOI: 10.1016/j.jns.2017.11.007.

Lazaridou A., Astrakas L., Mintzopoulos D., Khanchiceh A., Singhal A., Moskowitz M., Rosen B., Tzika A. 2013. fMRI as a molecular imaging procedure for the functional reorganization of motor systems in chronic stroke. Mol. Med. Rep. 3: 775-9. DOI: 10.3892/mmr.2013.1603.

Li B., Daunizeau J., Stephan K.E., Penny W., Hu D., Friston K. 2011. Generalised filtering and stochastic DCM for fMRI. Neuroimage 58: 442-57. DOI: 10.1016/j.neuroimage.2011.01.085.

Li S. 2017. Spasticity, Motor Recovery, and Neural Plasticity after Stroke. Front Neurol. 8: 120. DOI: $10.3389 /$ fneur.2017.00120.

Ma L., Steinberg J.L., Cunningham K.A., Lane S.D., Kramer L.A., Narayana P.A., Kosten T.R., Bechara A., Moeller F.G. 2015. Inhibitory behavioral control: a stochastic dynamic causal modeling study using network discovery analysis. Brain Connect. 5: 177-86. DOI: 10.1089/brain.2014.0275.

Ma L., Steinberg J.L., Moeller F.G., Johns S.E., Narayana P.A. 2015 Effect of cocaine dependence on brain connections: clinical implications. Expert Rev Neurother. 15: 1307-19. DOI: $10.1586 / 14737175.2015 .1103183$. 
676 Mehta J.P., Verber M.D., Wieser J.A., Schmit B.D., Schindler-Ivens S.M. 2012. The effect of 677 movement rate and complexity on functional magnetic resonance signal change during pedaling.

678 Motor Control. 16: 158-75. DOI: 10.1123/mcj.16.2.158.

679

680 Moulton E., Galléa C., Kemlin C., Valabregue R., Maier M.A., Lindberg P., Rosso C. 2017.

681 Cerebello-Cortical Differences in Effective Connectivity of the Dominant and Non-dominant

682 Hand during a Visuomotor Paradigm of Grip Force Control. Front Hum Neurosci. 11: 511. DOI:

683 10.3389/fnhum.2017.00511.

684

685 Nowak D.A., Grefkes C., Dafotakis M., Eickhoff S., Küst J., Karbe H., Fink G.R. 2008. Effects 686 of low-frequency repetitive transcranial magnetic stimulation of the contralesional primary motor 687 cortex on movement kinematics and neural activity in subcortical stroke. Arch Neurol. 65: 741-7. 688 DOI: 10.1001/archneur.65.6.741.

689

690

Pavlova E.L., Semenov R.V., Guekht A.B. Effect of tDCS on Fine Motor Control of Patients in

691 Subacute and Chronic Post-Stroke Stages. 2019. J Mot Behav. 17: 1-13. DOI:

$69210.1080 / 00222895.2019 .1639608$.

693

694

Penfield W., Boldrey E. 1937. Somatic motor and sensory representation in the cerebral cortex of

695

696

697 Penny W.D., Stephan K.E., Daunizeau J., Rosa M.J., Friston K.J., Schofield T.M., Leff A.P.

698 2010. Comparing families of dynamic causal models. PLoS Comput. Biol. 6: e1000709. DOI:

699 10.1371/journal.pcbi.1000709.

700

701

Penny W.D., Stephan K.E., Mechelli A., Friston K.J. 2004. Comparing dynamic causal models.

702

Neuroimage 22:1157-72. DOI: 10.1016/j.neuroimage.2004.03.026.

703

704

705

Pool E.M., Rehme A.K., Fink G.R., Eickhoff S.B., Grefkes C. 2013. Network dynamics engaged 706

707

708

709

710

711

712 in the modulation of motor behavior in healthy subjects. Neuroimage 82: 68-76. DOI: 10.1016/j.neuroimage.2013.05.123.

713

714

Rehme A.K., Eickhoff S.B., Wang L.E., Fink G.R., Grefkes C. 2011. Dynamic causal modeling of cortical activity from the acute to the chronic stage after stroke. Neuroimage 55: 1147-58. DOI: 10.1016/j.neuroimage.2011.01.014.

715

Roc A.C., Wang J., Ances B.M., Liebeskind D.S., Kasner S.E., Detre J.A. 2006. Altered hemodynamics and regional cerebral blood flow in patients with hemodynamically significant stenoses. Stroke 37: 382-7. 
716 Röther J., Knab R., Hamzei F., Fiehler J., Reichenbach J.R., Büchel C., Weiller C. 2002.

717 Negative dip in BOLD fMRI is caused by blood flow oxygen consumption uncoupling in

718 humans. Neuroimage 15: 98-102.

719

720

Saleh S., Yarossi M., Manuweera T., Adamovich S., Tunik E. 2016. Network interactions

721 underlying mirror feedback in stroke: A dynamic causal modeling study. Neuroimage Clin. 13:

722

723

724 46-54. DOI: 10.1016/j.nicl.2016.11.012.

725

Seghier M.L., Zeidman P., Neufeld N.H., Leff A.P., Price C.J. 2010. Identifying abnormal

726 connectivity in patients using dynamic causal modeling of FMRI responses. Front Syst Neurosci. 4. pii: 142 . DOI: $10.3389 /$ fnsys. 2010.00142

727

728

729

730

731

732

733

734

Smith S.M. 2002. Fast robust automated brain extraction. Human Brain Mapp. 17: 143-155.

735

736

737

738

Stephan K.E., Penny W.D., Daunizeau J., Moran R.J., Friston K.J. 2009. Bayesian model selection for group studies. Neuroimage 46:1004-17. DOI: 10.1016/j.neuroimage.2009.03.025.

739

740

Stephan K.E., Weiskopf N., Drysdale P.M., Robinson P.A., Friston K.J. 2007. Comparing hemodynamic models with DCM. Neuroimage 38: 387-401. DOI:

10.1016/j.neuroimage.2007.07.040.

741

742

Tanji J., Shima K. 1994. Role for supplementary motor area cells in planning several movements ahead. Nature 371: 413-416.

van den Heuvel M.P., Hulshoff Pol H.E. 2010. Specific somatotopic organization of functional connections of the primary motor network during resting state. Hum. Brain Mapp. 31: 631-644. DOI: 10.1002/hbm.20893.

743

744 Vér C., Emri M., Spisák T., Berényi E., Kovács K., Katona P., Balkay L., Menyhárt L., Kardos 745 L., Csiba L. 2016. The Effect of Passive Movement for Paretic Ankle-Foot and Brain Activity in 746 Post-Stroke Patients. Eur. Neurol. 76: 132-142. DOI: 10.1159/000448033.

747

748 Vinehout K., Schmit B.D., Schindler-Ivens S. 2019. Lower Limb Task-Based Functional

749 Connectivity Is Altered in Stroke. Brain Connect. 9: 365-377. DOI: 10.1089/brain.2018.0640.

750

Volz L.J., Eickhoff S.B., Pool E.M., Fink G.R., Grefkes C. 2015. Differential modulation of

752 motor network connectivity during movements of the upper and lower limbs. Neuroimage 119: 44-53. DOI: 10.1016/j.neuroimage.2015.05.101. 
755 Wang L., Zhang J., Zhang Y., Yan R., Liu H., Qiu M. 2016. Conditional Granger Causality

756 Analysis of Effective Connectivity during Motor Imagery and Motor Execution in Stroke

757 Patients. Biomed Res Int. 3870863. DOI: 10.1155/2016/3870863.

758

759 Ward N.S., Brown M.M., Thompson A.J., Frackowiak R.S. 2003. Neural correlates of outcome 760 after stroke: a cross-sectional fMRI study. Brain 126: 1430-48.

761

762 Zeiler S.R., Krakauer J.W. 2013. The interaction between training and plasticity in the poststroke 763 brain. Curr Opin Neurol. 26: 609-16. DOI: 10.1097/WCO.0000000000000025. 


\section{Table $\mathbf{1}$ (on next page)}

Demographics, pathology data of stroke patients, clinical characteristics and NIHSS scores. 
1 Table 1. Demographics, pathology data of stroke patients, clinical characteristics and NIHSS scores.

\begin{tabular}{|c|c|c|c|c|c|}
\hline $\begin{array}{l}\text { Patient/ } \\
\text { Gender }\end{array}$ & $\begin{array}{l}\text { Age } \\
\text { (years) }\end{array}$ & Lesion type and topography (by CT) & Time of stroke & $\begin{array}{l}\text { Severity of lower limb } \\
\text { paresis }\end{array}$ & NIHSS \\
\hline 1/Male & 63 & Cerebral infarct in right MCA region & 1 Month & $\begin{array}{l}\text { Left sided mild- } \\
\text { moderate paresis }\end{array}$ & 1 \\
\hline 2/Female & 65 & Cerebral infarct in left MCA & 1 Month & $\begin{array}{l}\text { Right sided severe } \\
\text { paresis }\end{array}$ & 3 \\
\hline 3/Female & 62 & $\begin{array}{l}\text { Bilateral lacunar infarcts, no fresh } \\
\text { lesion }\end{array}$ & 1 Month & $\begin{array}{l}\text { Right sided moderate } \\
\text { paresis }\end{array}$ & 3 \\
\hline 4/Male & 56 & Cerebral infarct in right MCA region & 1 Month & $\begin{array}{l}\text { Left sided moderate } \\
\text { paresis }\end{array}$ & 3 \\
\hline 5/Male & 52 & No fresh lesion & 9 days & $\begin{array}{l}\text { Left sided moderate } \\
\text { paresis }\end{array}$ & 3 \\
\hline 6/Female & 82 & $\begin{array}{l}\text { Hypodens lesions in right } \\
\text { hemisphere }\end{array}$ & 6 days & $\begin{array}{l}\text { Left sided moderate } \\
\text { paresis }\end{array}$ & 2 \\
\hline 7/Female & 75 & $\begin{array}{l}\text { Cerebral infarct in left MCA, old } \\
\text { cerebral infarct in right MCA region }\end{array}$ & 12 days & Right sided mild paresis & 2 \\
\hline 8/Female & 71 & $\begin{array}{l}\text { Lesions frontal horns and cella } \\
\text { media in right hemisphere }\end{array}$ & 11 days & $\begin{array}{l}\text { Left sided moderate } \\
\text { paresis }\end{array}$ & 2 \\
\hline 9/Male & 59 & No fresh lesion & 5 days & Right sided mild paresis & 1 \\
\hline 10/Male & 58 & $\begin{array}{l}\text { No fresh lesion, old lesions in basal } \\
\text { ganglia }\end{array}$ & 9 days & $\begin{array}{l}\text { Left sided moderate } \\
\text { paresis }\end{array}$ & 2 \\
\hline
\end{tabular}

2 NIHSS: National Institutes of Health Stroke Scale 


\section{Table 2 (on next page)}

Definitions of mild and moderate paresis and severe paresis $\left(6^{\text {th }}\right.$ item of the National Institutes of Health Stroke Scale). 
1 Table 2. Definitions of mild and moderate paresis and severe paresis $\left(6^{\text {th }}\right.$ item of the National 2 Institutes of Health Stroke Scale)

3

\begin{tabular}{|l|l|l|}
\hline \multirow{2}{*}{$\begin{array}{l}\text { Definition of mild and } \\
\text { moderate paresis }\left(6^{\text {th }}\right. \\
\text { item of NIHSS) }\end{array}$} & 1 point & $\begin{array}{l}\text { Drift; leg falls by the end of the 5-second period but } \\
\text { does not hit the bed }\end{array}$ \\
\cline { 2 - 3 } & 2 points & $\begin{array}{l}\text { Some effort against gravity; leg falls to bed by } 5 \\
\text { seconds but has some effort against gravity }\end{array}$ \\
\hline $\begin{array}{l}\text { Definition of severe } \\
\text { paresis } \\
\left.6^{\text {th }} \text { item of NIHSS }\right)\end{array}$ & 3 points & No effort against gravity; leg falls to bed immediately \\
\cline { 2 - 3 } & 4 points & No movement \\
\hline
\end{tabular}

4 


\section{Table 3(on next page)}

Summarized statistical table of intrinsic (endogenous) connection strength analysis of the winning model performed by Monte-Carlo-based exact permutation tests following False Discovery Rate (FDR) correction. 
1 Table 3. Summarized statistical table of intrinsic (endogenous) connection strength analysis of the

2 winning model performed by Monte-Carlo-based exact permutation tests following False Discovery Rate

3 (FDR) correction.

\begin{tabular}{|c|c|c|c|c|c|c|}
\hline Connections & $\begin{array}{l}\text { Mean connection } \\
\text { strength during non- } \\
\text { paretic ankle } \\
\text { movement }\end{array}$ & $\begin{array}{l}\text { SD of connection } \\
\text { strengths during non- } \\
\text { paretic ankle } \\
\text { movement }\end{array}$ & $\begin{array}{l}\text { Mean connection } \\
\text { strength during } \\
\text { paretic ankle } \\
\text { movement }\end{array}$ & $\begin{array}{l}\text { SD of connection } \\
\text { strengths during } \\
\text { paretic ankle } \\
\text { movement }\end{array}$ & $\mathrm{p}$ value & $\begin{array}{c}\text { FDR } \\
\text { corrected } \mathrm{p} \\
\text { value }\end{array}$ \\
\hline cM1 $\rightarrow$ cM1 & -0.0925 & 0.0227 & -0.1389 & 0.0489 & 0.0119 & 0.0395 \\
\hline $\mathrm{cM} 1 \rightarrow \mathrm{cPM}$ & 0.0224 & 0.1058 & 0.1086 & 0.0742 & 0.0510 & 0.0756 \\
\hline $\mathrm{cM} 1 \rightarrow \mathrm{cSMA}$ & 0.0903 & 0.1060 & 0.0636 & 0.1118 & 0.5905 & 0.3992 \\
\hline $\mathrm{cM} 1 \rightarrow \mathrm{iM1}$ & 0.1649 & 0.0438 & 0.2651 & 0.1101 & 0.0127 & 0.0395 \\
\hline $\mathrm{cPM} \rightarrow \mathrm{cM} 1$ & 0.0174 & 0.0667 & 0.0648 & 0.0597 & 0.1069 & 0.1073 \\
\hline $\mathrm{cPM} \rightarrow \mathrm{cPM}$ & -0.1339 & 0.0295 & -0.1051 & 0.0388 & 0.0778 & 0.0939 \\
\hline $\mathrm{cPM} \rightarrow \mathrm{cSMA}$ & 0.1345 & 0.0896 & 0.0592 & 0.0671 & 0.0485 & 0.0744 \\
\hline $\mathrm{cS} 1 \rightarrow \mathrm{cM} 1$ & 0.03680 & 0.0361 & 0.0361 & 0.0514 & 0.9768 & 0.5236 \\
\hline $\mathrm{cS} 1 \rightarrow \mathrm{cPM}$ & -0.0168 & 0.0401 & -0.0064 & 0.0694 & 0.726 & 0.4496 \\
\hline $\mathrm{cS} 1 \rightarrow \mathrm{cS} 1$ & -0.1021 & 0.0281 & -0.1688 & 0.0579 & 0.0045 & 0.0395 \\
\hline cSMA $\rightarrow$ cM1 & 0.0886 & 0.0511 & $6.3100 \mathrm{e}-06$ & 0.0871 & 0.0123 & 0.0395 \\
\hline $\mathrm{cSMA} \rightarrow \mathrm{cPM}$ & 0.244 & 0.1376 & 0.1206 & 0.1464 & 0.0690 & 0.0887 \\
\hline cSMA $\rightarrow$ cSMA & -0.1780 & 0.0340 & -0.1350 & 0.0336 & 0.0133 & 0.0395 \\
\hline $\mathrm{cSMA} \rightarrow \mathrm{iSMA}$ & 0.2755 & 0.0797 & 0.3023 & 0.0896 & 0.4771 & 0.3493 \\
\hline $\mathrm{iM} 1 \rightarrow \mathrm{cM} 1$ & 0.1752 & 0.0834 & 0.2795 & 0.1319 & 0.0488 & 0.0746 \\
\hline $\mathrm{iM} 1 \rightarrow \mathrm{iM} 1$ & -0.1062 & 0.0287 & -0.1303 & 0.0413 & 0.1447 & 0.1400 \\
\hline $\mathrm{iM} 1 \rightarrow \mathrm{iPM}$ & -0.0003 & 0.1588 & 0.0155 & 0.1001 & 0.7919 & 0.4712 \\
\hline $\mathrm{iM} 1 \rightarrow \mathrm{iSMA}$ & 0.0727 & 0.0605 & 0.0932 & 0.0924 & 0.5682 & 0.3900 \\
\hline $\mathrm{iPM} \rightarrow \mathrm{iM} 1$ & -0.0024 & 0.1094 & -0.0237 & 0.0260 & 0.5641 & 0.3883 \\
\hline $\mathrm{iPM} \rightarrow \mathrm{iPM}$ & -0.1310 & 0.0517 & -0.1332 & 0.0544 & 0.9277 & 0.5107 \\
\hline $\mathrm{iPM} \rightarrow \mathrm{iSMA}$ & 0.0304 & 0.0757 & 0.1112 & 0.1245 & 0.0966 & 0.1031 \\
\hline $\mathrm{iS} 1 \rightarrow \mathrm{iM} 1$ & 0.0382 & 0.0849 & 0.0363 & 0.0716 & 0.9615 & 0.5197 \\
\hline $\mathrm{iS} 1 \rightarrow \mathrm{iPM}$ & -0.0414 & 0.1473 & -0.0286 & 0.0712 & 0.8337 & 0.4840 \\
\hline $\mathrm{iS} 1 \rightarrow \mathrm{iS} 1$ & -0.1870 & 0.0784 & -0.1391 & 0.0473 & 0.1042 & 0.1063 \\
\hline iSMA $\rightarrow$ cSMA & 0.3144 & 0.1231 & 0.2658 & 0.0669 & 0.2867 & 0.2439 \\
\hline iSMA $\rightarrow$ iM1 & 0.1054 & 0.0548 & 0.0262 & 0.0893 & 0.0274 & 0.0591 \\
\hline
\end{tabular}




\begin{tabular}{|c|c|c|c|c|c|c|}
\hline iSMA $\rightarrow$ iPM & 0.1260 & 0.0971 & 0.1808 & 0.1623 & 0.3723 & 0.2952 \\
\hline iSMA $\rightarrow$ iSMA & -0.1316 & 0.0337 & -0.1709 & 0.0393 & 0.0298 & 0.0615 \\
\hline $\begin{array}{l}\text { SD: standard de } \\
\text { p: probability } \\
\text { c: contralateral } \\
\text { i: ipsilateral } \\
\text { M1: primary mo } \\
\text { PM: premotor c } \\
\text { SMA: supplem } \\
\text { S1: primary son }\end{array}$ & & & & & & \\
\hline
\end{tabular}

12 


\section{Figure 1}

Model selection for motor network.

(A) Base model: two-way extrinsic (endogenous) connections between PM, SMA, and M1 regions in both hemispheres, and the non-paretic-side M1 and paretic-side M1 areas and nonparetic-side SMA and paretic-side SMA regions were connected. (B) Differences of Model variations organized by two families: The external inputs are shown in the rows, which was only considered on the contralateral side. The columns show the connection system between the S1 and the motor network. (C) An example of the combination of the base model and Model4. 

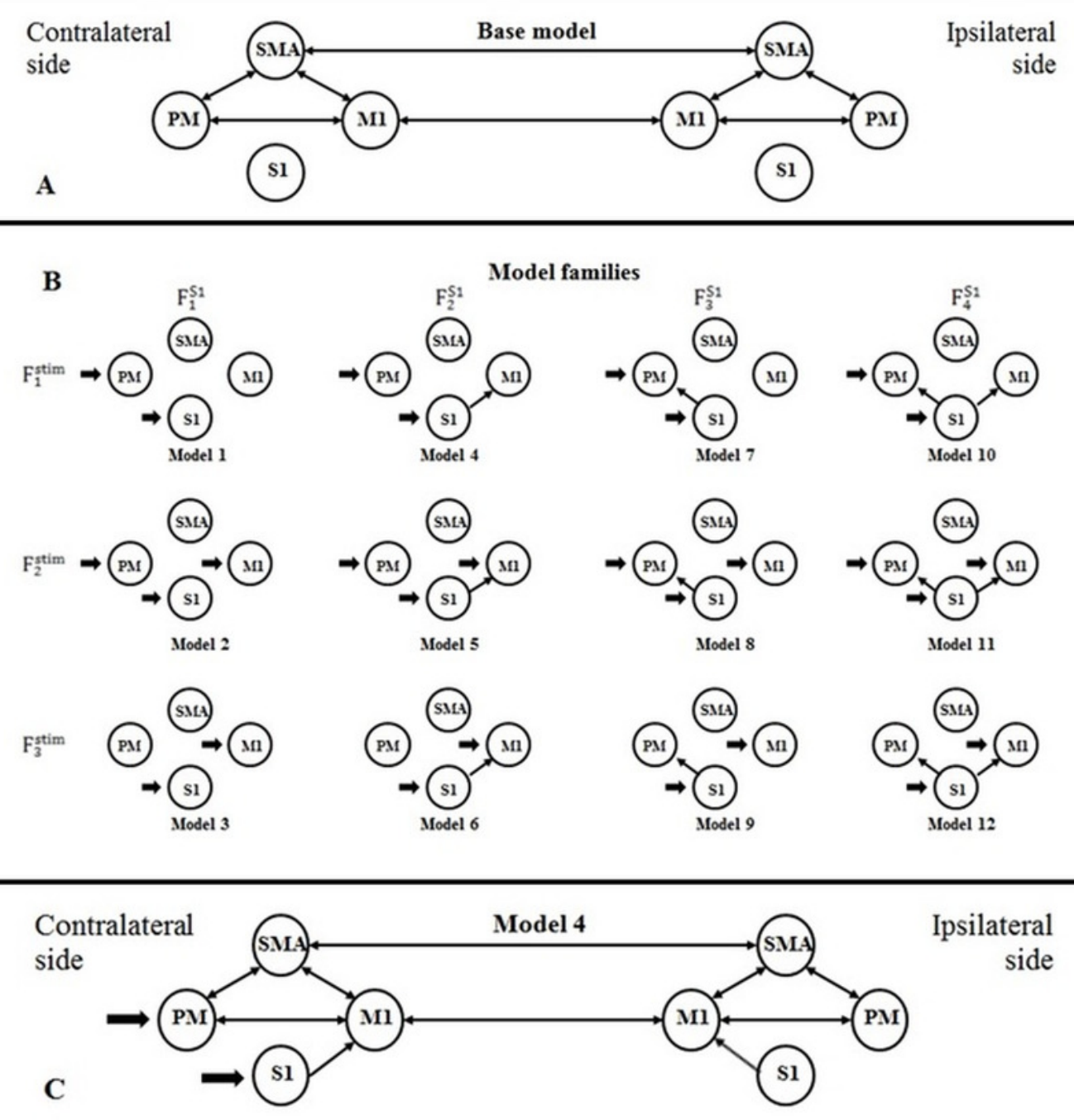


\section{Figure 2}

Results of the Bayesian Model Comparison (BMC) for the $\mathrm{F}^{51}$ and $\mathrm{F}^{\text {stim }}$ model family sets.

Results of the Bayesian Model Comparison (BMC) for the $\mathrm{F}^{\mathrm{s1}}$ and $\mathrm{F}^{\text {stim }}$ model family sets.

Models of both non-paretic and paretic ankle continuous passive movement (CPM) was

included in family-wise comparisons. The most probable network topology types were the $\mathrm{F}^{\mathrm{S1}}$ family, with expected probability (Exp_p) of 0.784 and exceedance probability (Exc_p) 0.998

(Panel A/B). For the direct effect model family selection, the $\mathrm{F}_{2}^{\text {stim }}{ }_{2}$ family was selected with 0.845 Exp_p and 0.999 Exc_p (Panel C/D). 
A

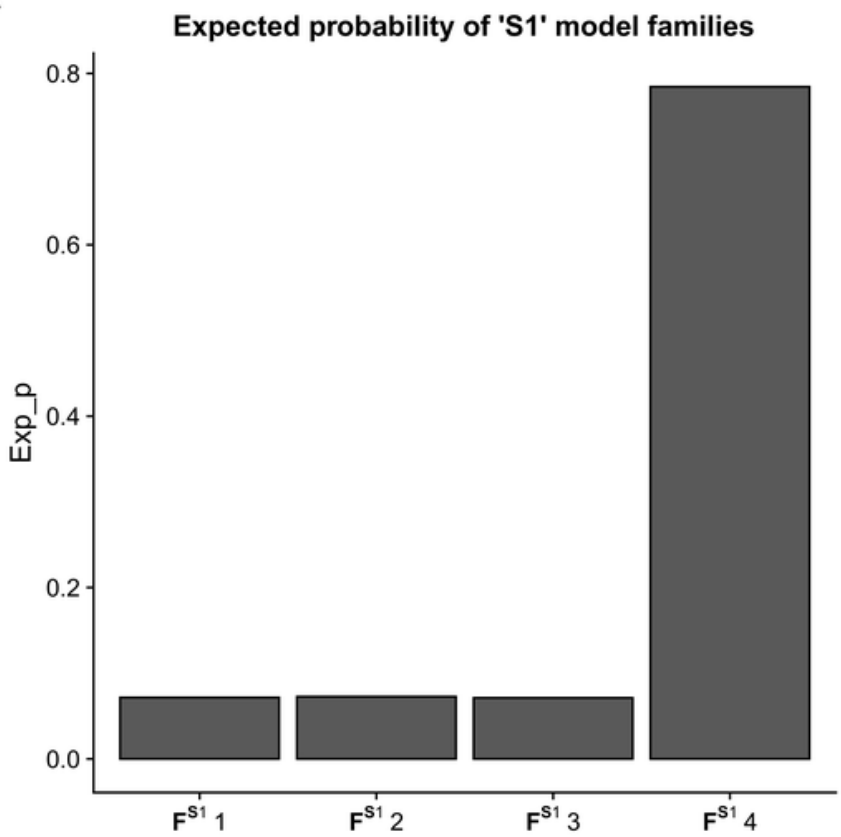

B

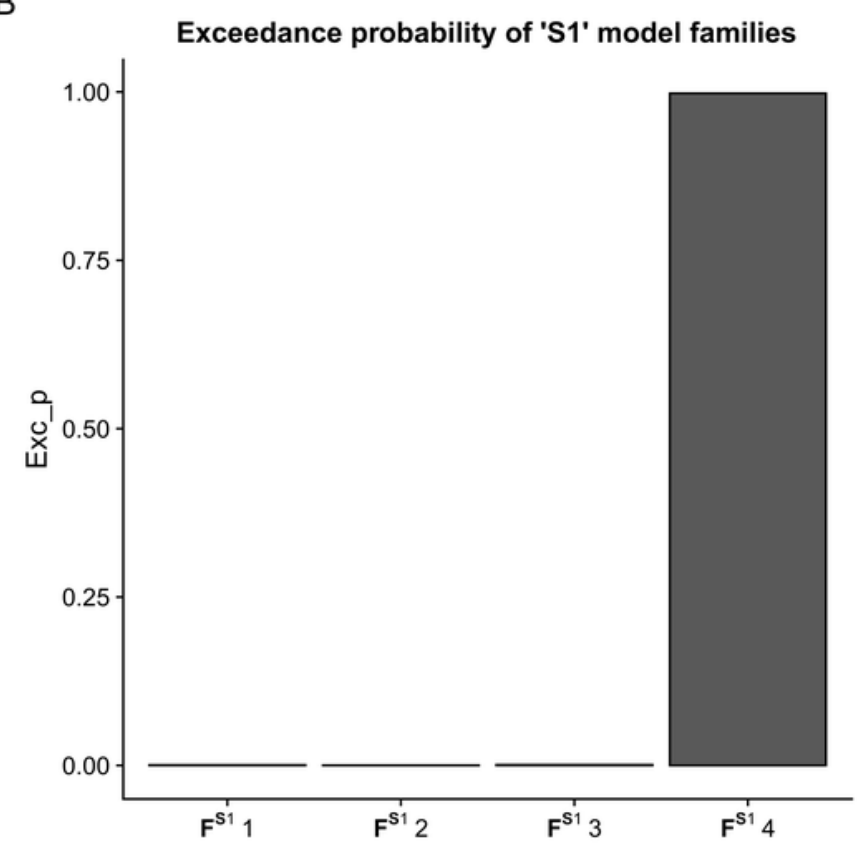

C

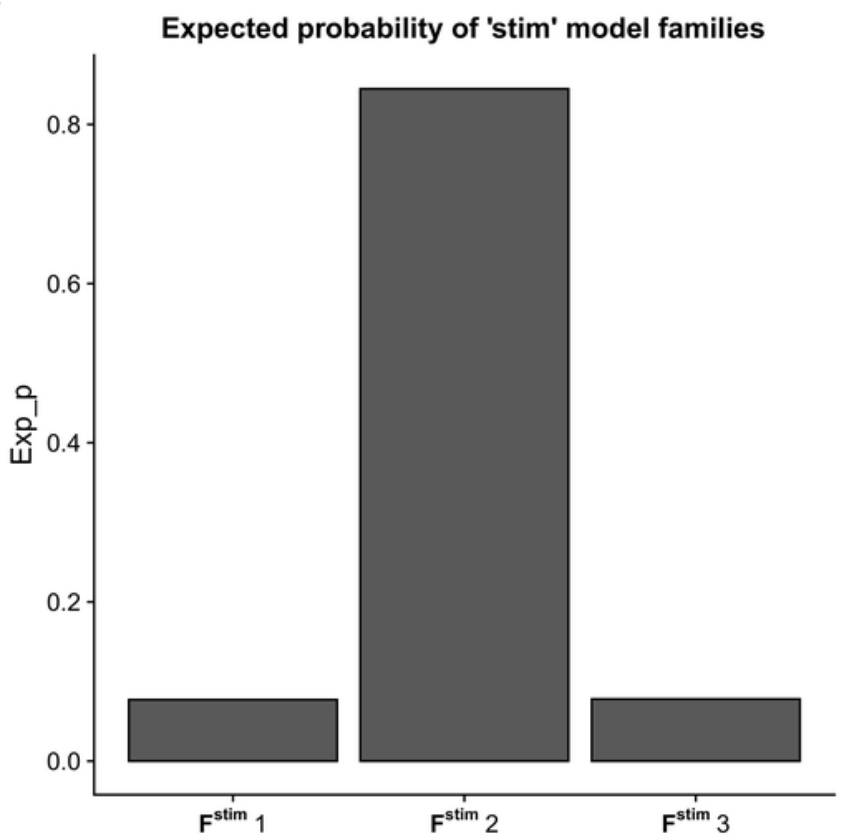

D

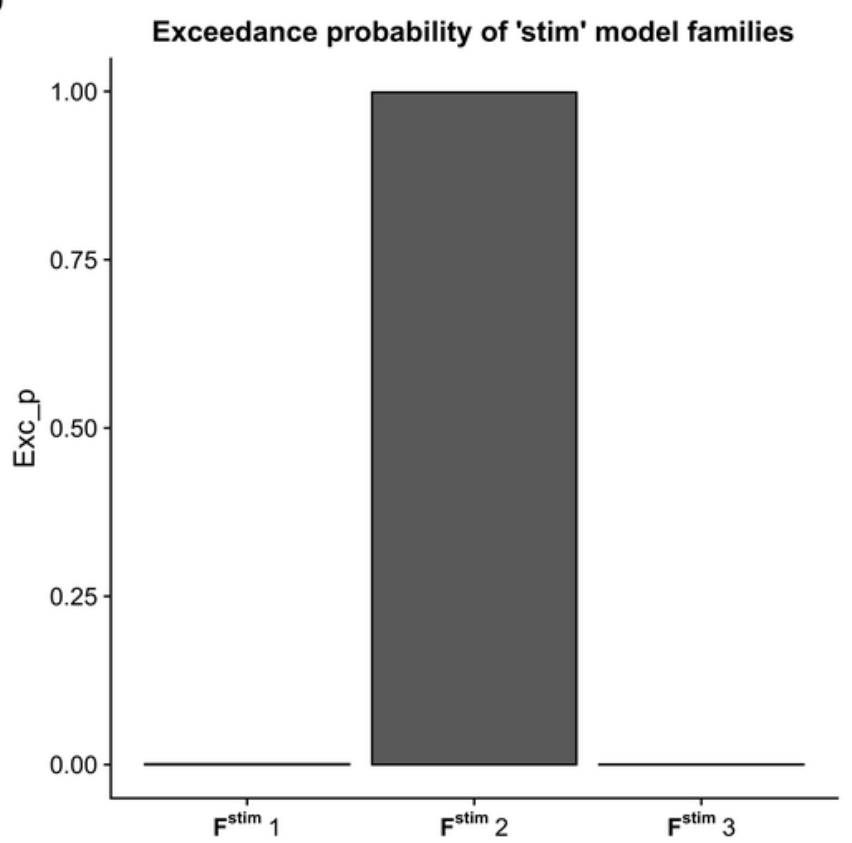




\section{Figure 3}

Comparison of model families.

Comparison of model families. (A) Base model: two-way extrinsic (endogenous) connections between PM, SMA, and M1 regions in both hemispheres, and the non-paretic-side M1 and paretic-side M1 areas and non-paretic-side SMA and paretic-side SMA regions were connected. (B) Panel B shows the 12 model variations organized in four $\mathrm{F}^{\mathrm{s1}}$ families (columns) and three $\mathrm{F}^{\text {stim }}$ families (rows). Based on BMC results, we found that these models have the most evidence with the highest expected probability that connects S1 to both M1 and PM cortices ( $\mathrm{F}_{4}^{\mathrm{S1}}$ denoted by - vertical grey rectangle). In the case of the direct effects of the stimulus changing between M1, PM, and S1 areas, the combination of all three regions provides the most probable pattern for stimulating the network for both the non-paretic and paretic sides ( $\mathrm{F}_{2}^{\text {stim }}{ }_{2}$ denoted by - horizontal grey rectangle). Based on this the best model is Model 11, indicated by a grey rectangle. 


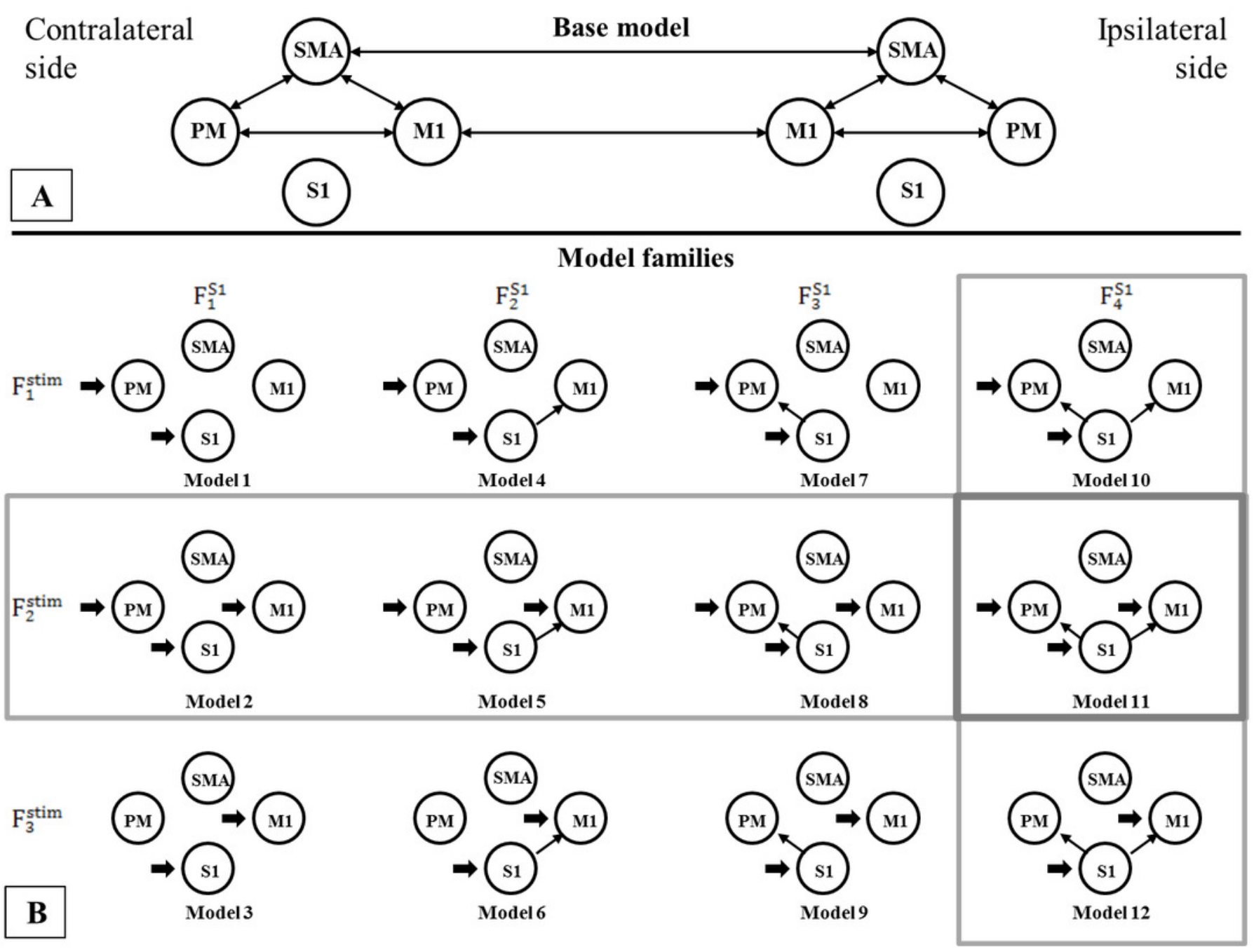




\section{Figure 4}

Mean values of significantly different endogenous connection (matrix A) strengths (see p-values in Table 3) of the best model (Model 11).

Bold arrows show the direction of significant connections. (A) Significant differences during non-paretic ankle movement: $\mathrm{CSMA} \rightarrow \mathrm{CSMA}$ (mean value $=-0.18$ ); $\mathrm{CSMA} \rightarrow \mathrm{CM} 1$ (mean value $=$ $0.08) ; \mathrm{CM} 1 \rightarrow \mathrm{CM} 1$ (mean value $=-0.09) ; \mathrm{CM} 1 \rightarrow \mathrm{iM} 1$ (mean value $=0.17)$ and $\mathrm{CS} 1 \rightarrow \mathrm{CS} 1$ (mean value $=-0.10$ ). (B) Significant differences during paretic ankle movement: CSMA $\rightarrow$ CSMA (mean value $=-0.13) ; \mathrm{CSMA} \rightarrow \mathrm{CM} 1$ (mean value $=-0.005) ; \mathrm{CM1} \rightarrow \mathrm{CM} 1$ (mean value $=-0.14)$; $c M 1 \rightarrow i M 1$ (mean value $=0.27$ ) and $\mathrm{CS} 1 \rightarrow \mathrm{CS} 1$ (mean value $=-0.16$ ). The empty arrows show the target location of external stimulus. The looping arrows represent the self-inhibitory effects. 


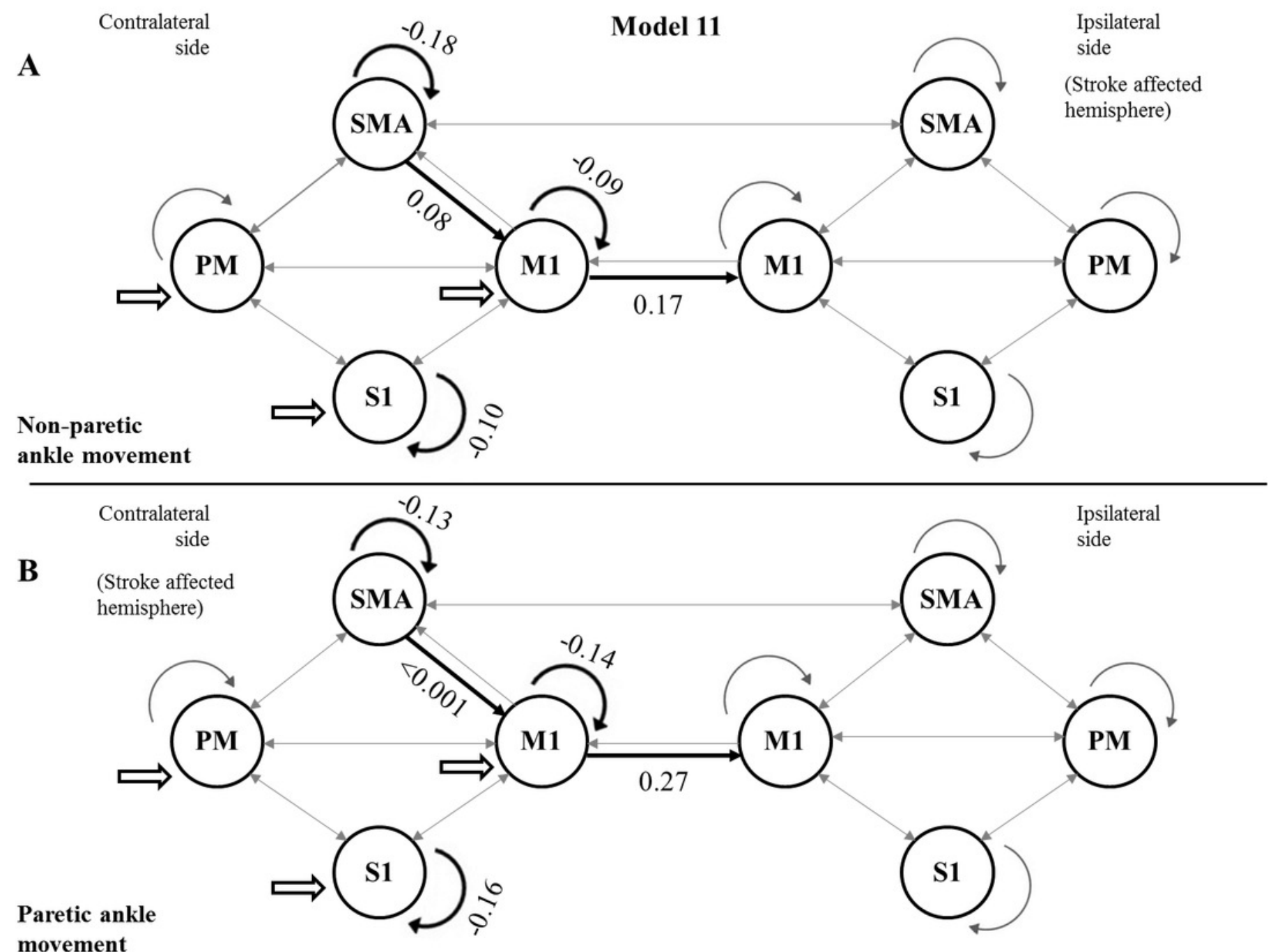




\section{Figure 5}

Comparison of mean values.

On the top of the images can be seen the FDR corrected $p$ value differences between paretic and non-paretic ankle movement. (A) The paretic CPM caused stronger self-inhibition in CM1. (B) The paretic CPM caused stronger self-inhibition in CS1. (C) The paretic CPM caused weaker self-inhibition in SMA. (D) The cSMA $\rightarrow$ CM1 connection changed and, the cSMA excited the neural activity of $\mathrm{CM} 1$. (E) The $\mathrm{CM} 1 \rightarrow \mathrm{M} 1 \mathrm{l}$ connection showed stronger excitation during the paretic CPM.

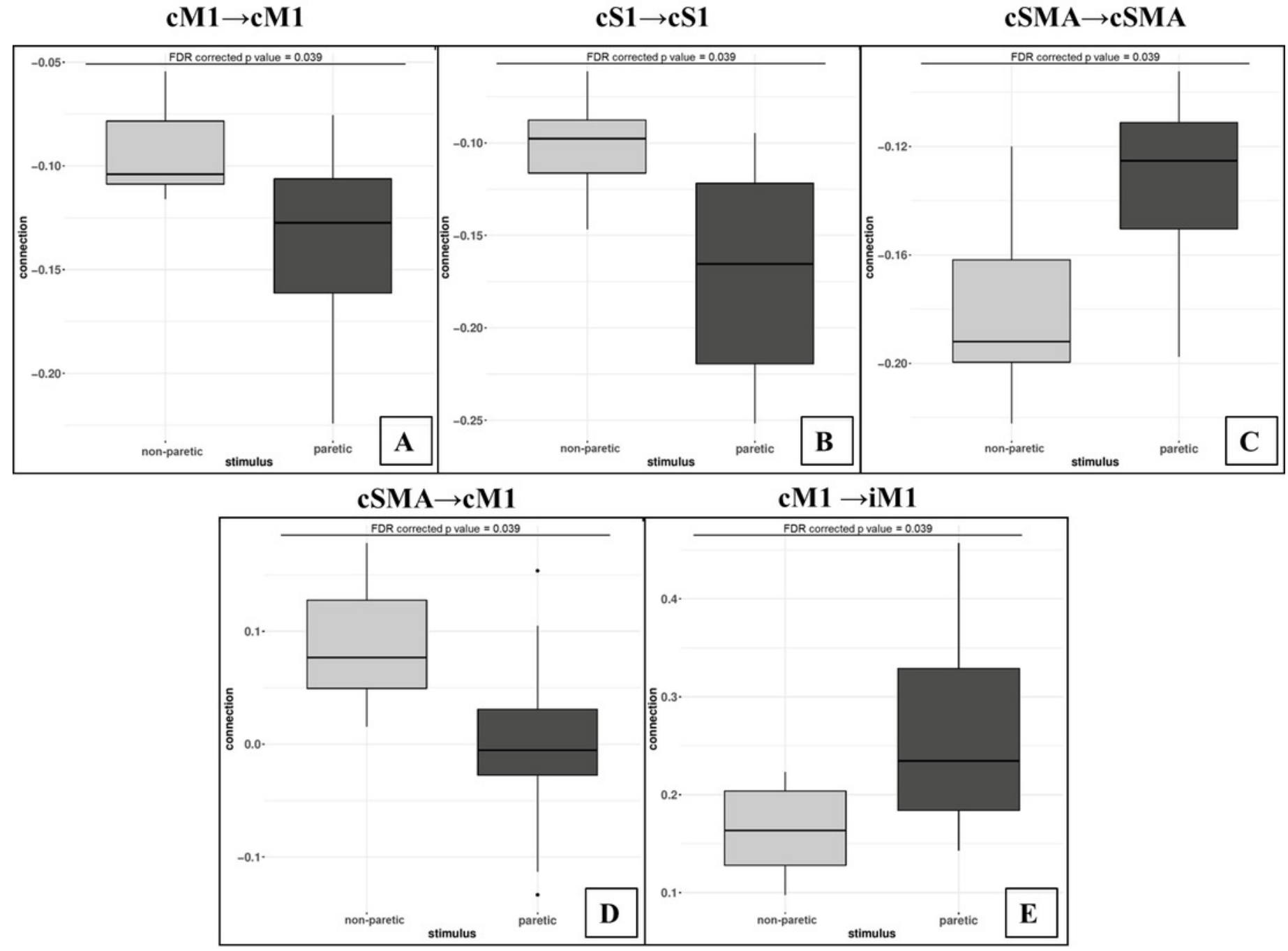

